\title{
KINETOPLASTID PHYLOGENETICS, WITH SPECIAL REFERENCE TO THE EVOLUTION OF PARASITIC TRYPANOSOMES
}

\author{
STEVENS J.R.*
}

\section{Summary:}

To fully understand the evolutionary history of parasitic kinetoplastids and to understand the context within which the evolution of each parasite group has developed, an understanding not just of the parasites, but of all kinetoplastids is required. Accordingly, this paper provides an overview of kinetoplastid evolution and systematics, including coverage of the proposal by Moreira et al. (2004) to divide kinetoplasts into Prokinetoplastina (Ichthyobodo and Perkinsiella) and Metakinetoplastina (other bodonids and trypanosomatids). The implications of such a revision, with regard to correctly identifying outgroup taxa for studies of evolution within taxa of medical importance, are addressed, together with a more detailed review of the evolution and origins of the trypanosomes in the light of new phylogenies, new approaches and revisions in kinetoplastid systematics.

KEY WORDS : kinetoplastid, trypanosome, Trypanosomatidae, Bodonidae, Prokinetoplastina, Metakinetoplastina, evolution.

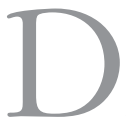
ue, in large part, to the particular focus of the various European colonial medical services in Africa, Asia and South America in the late 1800s and early 1900s (Castellani, 1903; Bruce \& Nabarro, 1903; Lyons, 1992), haemoflagellate parasites of humans and domestic animals, including trypanosomes and Leishmania spp. are among some of the most wellstudied agents of parasitic diseases known today. In consequence, some of these organisms have long been the subject of investigation and speculation with regard to their evolutionary history (Léger, 1904; Wallace, 1966; Hoare, 1972; Vickerman, 1994) and, indeed, trypanosomatids were among some of the first parasites to be characterised with the new methods of isoenzyme typing developed in the 1970s (Godfrey \& Kilgour, 1976; Miles et al., 1977). For many kinetoplastid groups (notably those of medical importance), the findings of early studies have since been superseded by results from more recent studies, which, in general, due to the techniques used, e.g. DNA sequencing and genomic analyses, have provided increased levels of resolution, robustness and accuracy. However, the application of new methodologies across all kinetoplastid groupings has been by no means equal (Podlipaev,

* School of Biosciences, University of Exeter, Exeter EX4 4PS, UK. E-mail: j.r.stevens@ex.ac.uk
2000) and even in those parasites of medical importance, it is little more than a decade since the widespread introduction of DNA sequencing technologies has allowed the evolution of parasitic trypanosomatids (and kinetoplastids in general) to be evaluated by formal phylogenetic analysis (Fernandes et al., 1993; Alvarez et al., 1996; Maslov et al., 1996; Lukes et al., 1997; Haag et al., 1998; Hannaert et al., 1998; Stevens et al., 1999, 2001; Wright et al., 1999; Hamilton et al., 2004; Simpson et al., 2004; Moreira et al., 2004).

To fully understand the evolutionary history of parasitic kinetoplastids and to understand the context within which the evolution of each parasite group has unfolded, an understanding not just of the parasites, but also of broader kinetoplastid phylogenetics is required. Accordingly, this paper provides an overview of kinetoplastid phylogenetics and evolution, together with a more detailed review of the evolution of the trypanosomes in the light of recent revisions in kinetoplastid systematics. For more comprehensive details of these topics, in addition to the references given above, readers are referred to reviews by Maslov et al. (2001), Stevens et al. (2001) and, most recently, Simpson et al. (2006).

\section{KINTEOPLASTID PHYLOGENETICS}

The order Kinetoplastida comprises a group of protozoa defined by the presence of a characteristic organelle, the kinetoplast (Vickerman, 1976), a large modified mitochondrion. Traditionally, the taxonomy of kinetoplastids has been based on morphological characters and lifecycles, with the group being subdivided into two suborders: Bodonina and Trypanosomatina (Vickerman, 1976; Lom, 1976). See Maslov et al. (2001) for an overview of this topic.

Briefly, suborder Bodonina included two families: Bodonidae and Cryptobiidae. Bodonids show a variety of life styles, ranging from free living, e.g. Bodo, Dimastigella, Rhynchobodo, to parasitic; the parasitic Bodonidae are represented by ectoparasites of fish, e.g. Ichthyobodo, and endoparasites of fish and snails (Cryptobia and Trypanoplasma, the latter being transmitted by 
leeches). The second group within Kinetoplastida, the trypanosomatids, comprise a single family, Trypanosomatidae; among others, this family includes the obligate parasites of medical and veterinary importance, trypanosomes and Leishmania sp., which have become the focus of so much research. Trypanosomatids infect all classes of vertebrates, together with some invertebrates, and also plants. Indeed, many invertebrates, e.g. tsetse flies, sandflies, triatomine bugs, are perhaps best known for their role as vectors for those disease agents with a digenetic lifecycle, e.g. trypanosomes, Leishmania sp. and Phytomonas sp. However, there are also many monogenetic parasites, e.g. Crithidia, Leptomonas, Herpetomonas and Blastocrithidia, which, while less well studied than their digenetic counterparts are equally as important from the perspective of understanding the evolution of the kinetoplastids as a whole.
As stated, this system of classifcation, which has been in place since the 1970s, was established based largely on morphological and lifecycle characters, in the absence of molecular data. More recently, however, the accumulation of much new molecular data from a diverse range of studies has increasingly suggested that the division of Kinetoplastida into the two suborders outlined above is artificial (Fig. 1), prompting Moreira et al. (2004) to propose a revision of the group. Briefly, the new system of Moreira et al. (2004) divides kinetoplasts into Prokinetoplastina (Ichthyobodo and Perkinsiella) and Metakinetoplastina (other bodonids and trypanosomatids). See Simpson et al. (2006) for full details regarding the broader significance of this major systematic revision. Such a revision, however, is important not just from a taxonomic perspective, but also from the point of view of identifying correct outgroup

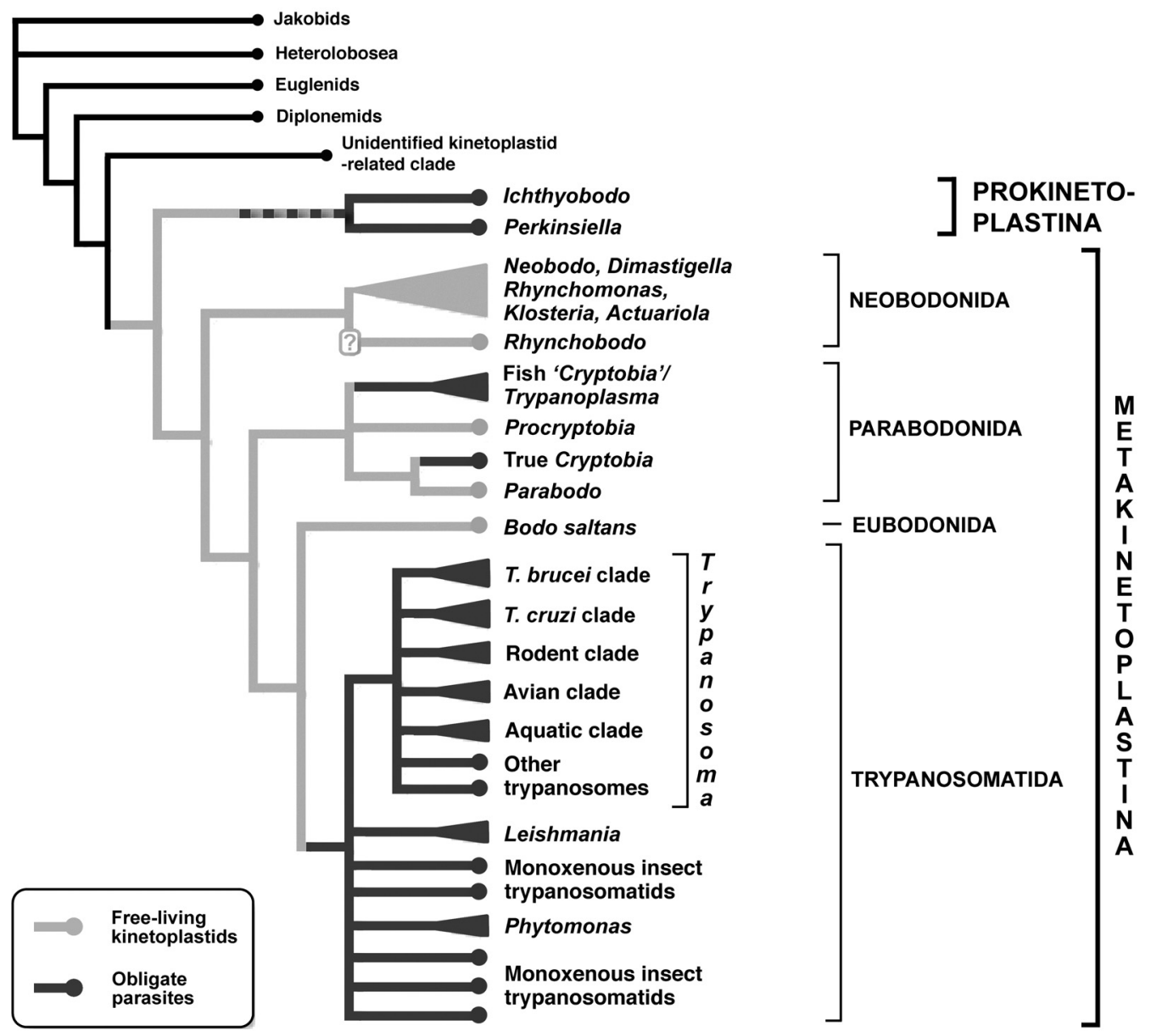

Fig. 1. - Evolutionary relationships among kinetoplastids based on recent taxon-rich SSU rRNA gene trees and protein phylogenies. The higher-level classification of kinetoplastids that was introduced by Moreira et al. (2004) is used. The placement of trypanosomatids as a sister group to Eubodonida is based on evidence from HSP phylogenies (Simpson \& Roger, 2004; Simpson et al., 2004). The "unidentified kinetoplastid-related clade" is known only from environmental SSU rRNA gene sequences (López-García et al., 2003); the organismal identity is not yet known. SSU rRNA and HSP analyses differ as to whether Rhynchobodo forms a specific clade with other neobodonids (Simpson et al., 2002). Black lines represent branches outside the kinetoplastid group; dark grey and clear grey lines indicates unknown status; circles denote single or a few known representatives of a particular clade; triangles denote several known representatives of a particular clade; question mark represents unstable clade position. Reproduced with permission from Trends in Parasitology; see Simpson et al. (2006) for full details. 
taxa for subsequent studies of evolution within, for example, groups of medical importance, a topic covered in more detail in the remainder of this paper.

\section{PHYLOGENETICS OF TRYPANOSOMES}

T wo issues dominate discussion of trypanosome evolution. Firstly, whether or not trypanosomes are monophyletic and, secondly, what gave rise to them. A monophyletic group consists of taxa that are descended from a single common ancestor and contains all known descendants from that ancestor. The first molecular phylogenetic studies, based on comparisons of mitochondrial and nuclear ribosomal DNAs (rDNA), showed trypanosomes to be paraphyletic (Gomez et al., 1991; Fernandes et al., 1993; Maslov et al., 1996), while subsequent rDNA-based studies, which have included more taxa from a far broader range of host species (Lukes et al., 1997; Stevens et al., 1999, 2001; Wright et al., 1999; Simpson et al., 2002; Hamilton et al., 2004) (Fig. 2) supported monophyly. However, Hamilton et al. (2004) also demonstrated that different large (> 68 taxa) 18S rDNA sequence alignments can either strongly support or reject monophyly depending on the range of taxa and the characters included in each analysis, so, while trypanosome monophyly appears highly probable, no definitive conclusion can be reached using this gene. Confidence in $18 \mathrm{~S}$ rDNA sequence data to infer phylogenetic relationships has also been challenged by the observation of two distinct copies of these genes in T. cruzi and other trypanosomatids (Stothard et al., 1998).

In contrast, phylogenetic studies based on proteincoding genes (Hannaert et al., 1992, 1998; Hashimoto et al., 1995; Adjé et al., 1998; Simpson et al., 2002) have consistently and unequivocally supported monophyly, a conclusion strongly supported by Hamilton et al. (2004) in their recent analyses of both glycosomal glyceraldehyde phosphate dehydrogenase (gGAPDH) DNA and protein trees from a broad range of trypanosomatid taxa, rooted using Euglena gracilis, an unequivocal outgroup taxon. Trypanosome monophyly is further supported by the phylogenies of Simpson et al. (2002) based on heat shock protein 90 amino acid sequences that include a different range of kinetoplastid taxa to the gGAPDH gene trees.

\section{THE ORIGIN OF TRYPANOSOMES}

A 11 trypanosomes are vertebrate blood parasites. Thus, taken together, the weight of evidence from this broad range of studies conducted to date suggests that trypanosomes are monophyletic and thus had a single evolutionary origin (Figs 2 and 3). From a single origin, trypanosomes appear to have radiated to infect all vertebrate classes, transmitted by a wide variety of blood-sucking vectors, including both terrestrial and aquatic leeches. The relationships of Leishmania and Endotrypanum, which are also vertebrate parasites, suggest that they do not share a common ancestor with trypanosomes and instead evolved from an insect-only trypanosomatid(s) comparatively recently. It is clear that $T$. bruce $i, T$. cruzi and Leishmania major, for which complete genome sequences are now available, are only distantly related and have distinct evolutionary histories. In view of the results presented here, comparison of trypanosome genomes may reveal ancestral genetic mechanisms for survival in vertebrates, which will not be shared by Leishmania. On the other hand, these trypanosomatids share common ancestral mechanisms for survival within their insect vectors.

A second implication of monophyly is that trypanosomes did not give rise to other trypanosomatid lineages by, for example, adapting to an invertebrateonly form of parasitism. This is surprising, as many trypanosome species undergo varied and complex life cycles in their invertebrate vectors. Instead, some trypanosomes have partly or completely lost dependency on the invertebrate host. For example, although usually transmitted by bloodsucking triatomine bugs, T. cruzi can be transmitted during breast-feeding (Miles, 1979) and T. equiperdum is transmitted during coitus between equines (Hoare, 1972).

Evidence from the recent broad gGAPDH gene phylogeny of Hamilton et al. (2004) also indicates that the trypanosome clade arises from within the wider group of trypanosomatids, which are parasites of insects (some of which have an additional vertebrate or plant host). Thus, the most likely ancestor of extant trypanosomes was an insect trypanosomatid parasite. There is further support for the insect parasite origin of genus Trypanosoma from the phylogenetic position of the leechtransmitted trypanosomes of fish and amphibia. If leechtransmitted trypanosomes of fish were the first to evolve as has been suggested (Woo, 1970; Baker, 1994; Vickerman, 1994), we would expect them to branch paraphyletically at the base of the trypanosome clade. This is not the case: the phylogenies presented here show all fish trypanosomes in a single, well-supported clade. In gGAPDH gene and most published $18 \mathrm{~S}$ rDNA trees, the fish trypanosome clade falls unequivocally in the "aquatic clade", which includes all aquatic leech-transmitted trypanosomes. Although we have no evidence whether the ancestor of the 'aquatic clade' trypanosomes was leech or insect-transmitted, as suggested by Maslov et al. (1996) it is clear that the majority of insect-transmitted trypanosomes did not evolve from this clade. 


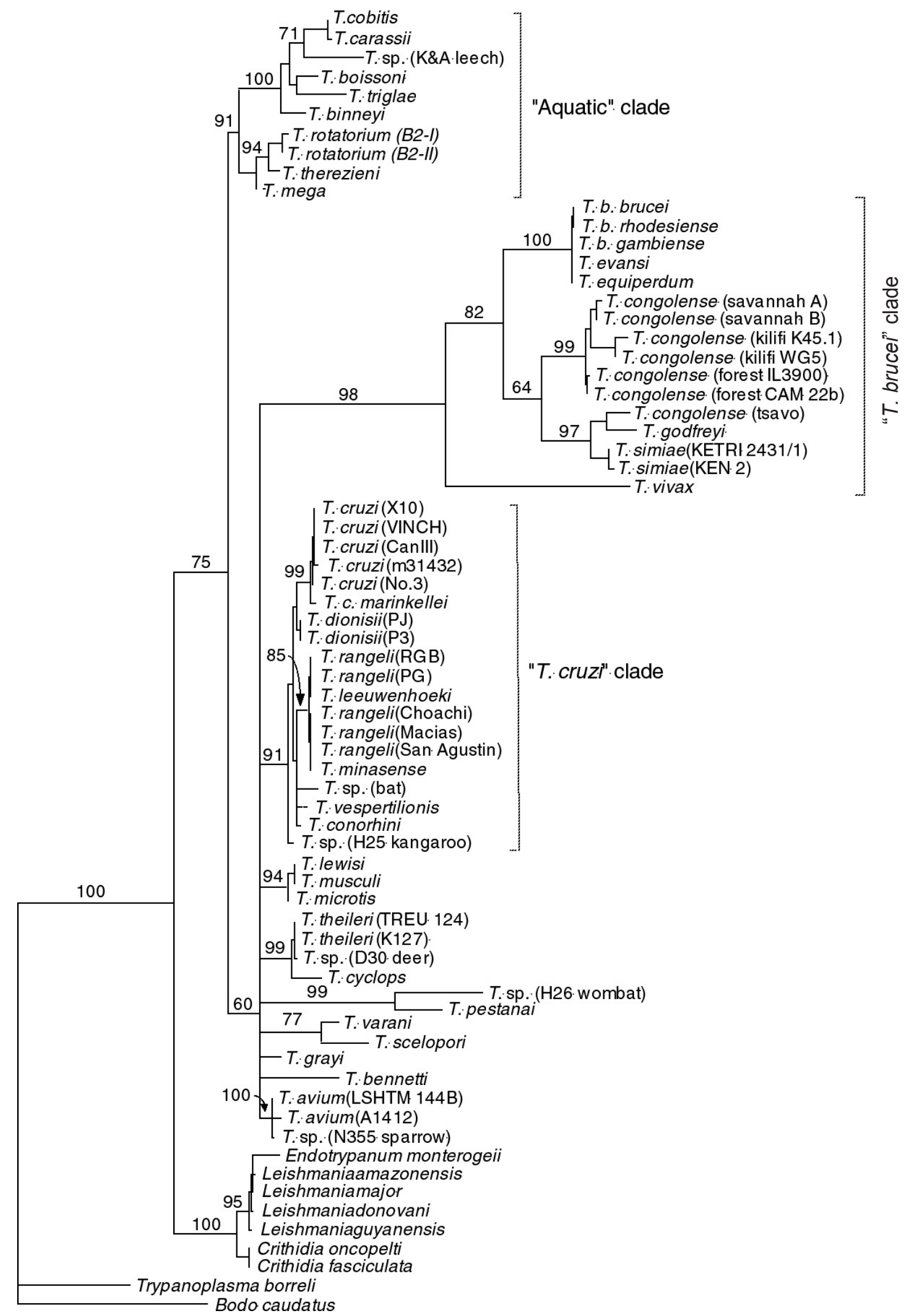

Fig. 2. - Small subunit ribosomal RNA-based gene tree calculated by bootstrapped maximum parsimony analysis. The tree represents an extended analysis of Stevens et al. (1999) and is based on an alignment of 1809 nucleotide positions. The phylogeny contains 61 Trypanosoma taxa and shows the genus to be monophyletic. T. congolense subgroups are denoted by $\mathrm{s}=$ savannah, $\mathrm{f}=\mathrm{forest}, \mathrm{k}=\mathrm{kilifi}$ and $\mathrm{t}=\mathrm{tsavo}$; sequence accession numbers are given in Haag et al. (1998) and Stevens et al. (1999, 2001). Analysis was perfomed using PAUP* 4 (Swofford, 1998). Reproduced with permission from Advances in Parasitology; see Stevens et al. (2001) for full details. 


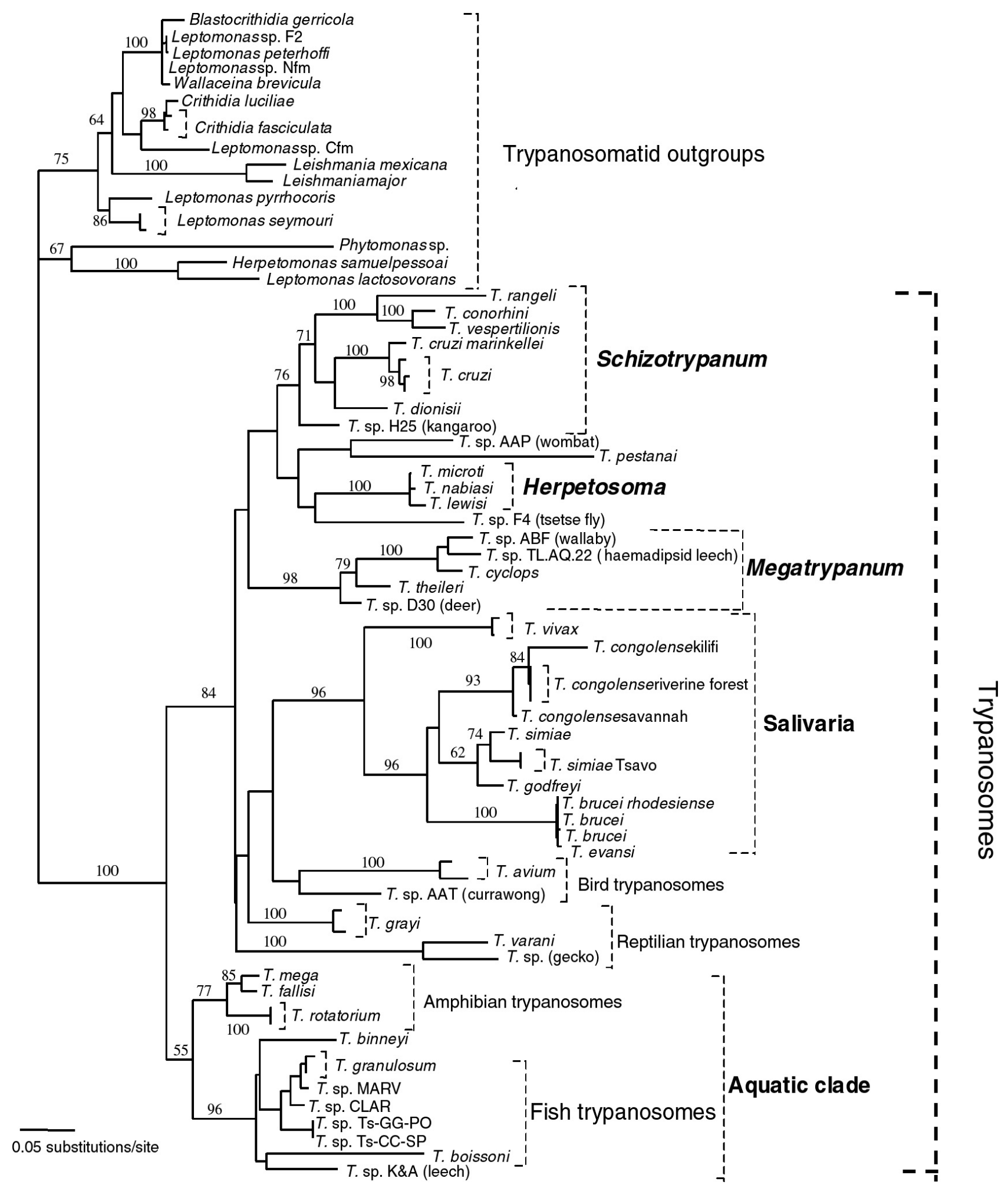

Fig. 3. - Maximum-likelihood tree constructed using PAUP* (Swofford, 2000) based on an alignment of 853 characters of the gGAPDH gene, including 72 taxa and rooted using non-trypanosome trypanosomatids; - $\ln$ L 12979.38. Values at nodes are ML bootstrap values (\%, 100 replicates). Redrawn from Hamilton et al. (2005); see Hamilton et al. (2004, 2005) for full details. 
Taken together, this evidence suggests a scenario whereby the ancestral trypanosome was an insect parasite. One of these early lineages, perhaps a parasite of a bloodsucking insect, adapted to and became dependant on vertebrate parasitism, giving rise to trypanosomes, as previously suggested (Hoare, 1972). The switch from an insect-only to an insect-transmitted trypanosome would have been a terrestrial event, assuming that, as today, extinct blood-sucking insects fed on land vertebrates. There are no reliable dates for the origins of most extant groups of blood-sucking insects; the earliest date is approximately 370 million years ago after the first land vertebrates appeared (Kardong, 2002). Intriguingly, $T$. binneyi, the trypanosome of the amphibious platypus, appears in the "aquatic clade"; this is a large trypanosome, similar in morphology to the trypanosomes of fish and amphibia Mackerras, (1959). It is thus plausible that the trypanosome species found in this primitive mammal has been acquired from aquatic leeches carrying fish trypanosomes (Stevens et al., 2001). Thus trypanosomes have not strictly coevolved with their vertebrate hosts; instead, a key factor in the evolutionary radiation of trypanosomes appears to have been transmission between vertebrates in shared environments (Stevens et al., 2001; Hamilton et al., 2007).

\section{ACKNOWLEDGEMENTS}

I would like to thank the many colleagues who have contributed to the ideas and hypotheses presented in this paper, some over the course of many years; chief among these are Patrick Hamilton, Wendy Gibson, David Godfrey, Julius Lukes, Alastair Simpson, Sergei Podlipaev, Harry Noyes, Chris Schofield, Sylvain Brisse, Michel Tibayrenc and John Baker. I would like to dedicate this paper to the memory of David Godfrey and Sergei Podlipaev, two often understated scientists who made important contributions to different parts of this fascinating field of parasite evolution.

\section{REFERENCES}

Adjé C.A., Opperdoes F.R. \& Michels P.A.M. Molecular analysis of phosphoglycerate kinase in Trypanoplasma borreli and the evolution of this enzyme in Kinetoplastida. Gene, 1998, 217, 91-99.

Alvarez F., Cortinas M.N. \& Musto H. The analysis of protein coding genes suggests monophyly of Trypanosoma. Molecular Phylogenetics and Evolution, 1996, 5, 333-343.

BAKER J.R. The origins of parasitism in the protists. International Journal for Parasitology, 1994, 24, 1131-1137.

Bruce D. \& Nabarro D. Progress report on Sleeping Sick- ness in Uganda. Report of the Sleeping Sickness Commission of the Royal Society, 1903, 1, 11-88.

CAstellani A. Presence of Trypanosoma in sleeping sickness. Report of the Sleeping Sickness Commission of the Royal Society, 1903, 1, 3-10.

Fernandes A.P., Nelson K. \& Beverley S.M. Evolution of nuclear ribosomal RNAs in kinetoplastid protozoa: perspectives on the age and origins of parasitism. Proceedings of the National Academy of Sciences of the USA, 1993, 90, 11608-11612.

Godfrey D.G. \& Kilgour V. Enzyme electrophoresis in characterizing the causative organism of Gambian trypanosomiasis. Transaction of the Royal Society of Tropical Medicine and Hygiene, 1976, 70, 219-224.

Gomez E., Valdes A.M., Pinero D. \& Hernandez R. What is a genus in the trypanosomatidae family - phylogenetic analysis of 2 small ribosomal-RNA sequences. Molecular Biology and Evolution, 1991, 8, 254-259.

HaAg J., O'Huigin C. \& Overath P. The molecular phylogeny of trypanosomes: evidence for an early divergence of the Salivaria. Molecular and Biochemical Parasitology, 1998, 91, 37-49.

Hamilton P.B., Stevens J.R., Gaunt M.W., Gidley J. \& Gibson W.C. Trypanosomes are monophyletic: evidence from genes for glyceraldehyde phosphate dehydrogenase and small subunit ribosomal RNA. International Journal for Parasitology, 2004, 34, 1393-1404.

Hamilton P.B., Stevens J.R., Gidley J., Holz P. \& Gibson W.C. A new lineage of trypanosomes from Australian vertebrates and terrestrial bloodsucking leeches (Haemadipsidae). International Journal for Parasitology, 2005, 35, 431-443.

Hamilton P.B., Gibson W.C. \& Stevens J.R. Patterns of co-evolution between trypanosomes and their hosts deduced from ribosomal RNA and protein-coding gene phylogenies. Molecular Phylogenetics and Evolution, 2007, 44, 15-25.

Hannaert V., Blaauw M., Kohl L., Allert S., Opperdoes F.R. \& Michels P.A. Molecular analysis of the cytosolic and glycosomal glyceraldehyde-3-phosphate dehydrogenase in Leishmania mexicana. Molecular and Biochemical Parasitology, 1992, 55, 115-126.

Hannaert V., Opperdoes F.R. \& Michels P.A.M. Comparison and evolutionary analysis of the glycosomal glyceraldehyde-3-phosphate dehydrogenase from different kinetoplastida. Journal of Molecular Evolution, 1998, 47, 728-738.

Hashimoto T., Nakamura Y., Kamaishi T., Adachi J., Nakamura F., Okamoto K. \& Hasegawa M. Phylogenetic place of kinetoplastid protozoa inferred from a protein phylogeny of elongation factor $1 \alpha$. Molecular and Biochemical Parasitology, 1995, 70, 181-185.

HoAre C.A. The trypanosomes of mammals. Blackwell Scientific Publications, Oxford, 1972, p. 749.

Kardong K.V. Vertebrates: Comparative Anatomy, Function, Evolution. McGraw-Hill Companies, Inc, New York, 2002.

LÉGER L. Surles affinites de l'Herpetomonas subulata et la phylogene des trypanosomes. Comp. Rend. Seances Soc. Biol. Ses. Fil., 1904, 56, 615-617.

Lom J. Biology of the trypanosomes and trypanoplasms of fish, in: Biology of the Kinetoplastida. Lumsden W.H.R. 
\& Evans D.A. (Eds), Academic Press, London/ New York/San Francisco, 1976, 269-337.

López-García P., Philippe H., Gaill F. \& Moreira D. Autochthonous eukaryotic diversity in hydrothermal sediment and experimental microcolonizers at the Mid-Atlantic Ridge. Proc. Natl. Acad. Sci. USA, 2003, 100, 697-702.

Lukes J., Jirku M., Dolezel D., Kral'ova I., Hollar L. \& Maslov D.A. Analysis of ribosomal RNA genes suggests that trypanosomes are monophyletic. Journal of Molecular Evolution, 1997, 44, 521-527.

Lyons M. The colonial disease. A social history of sleeping sickness in northern Zaire, 1900-1940. Cambridge University Press, Cambridge, 1992, p. 335.

Mackerras M.J. The haematozoa of Australian mammals. Australian Journal of Zoology, 1959, 7, 105-135.

Maslov D.A., Lukes J., JiRku M. \& Simpson L. Phylogeny of trypanosomes as inferred from the small and large subunit rRNAs: implications for the evolution of parasitism in the trypanosomatid protozoa. Molecular and Biochemical Parasitology, 1996, 75, 197-205.

Maslov D.A., Podlipaev S.A. \& Lukes J. Phylogeny of the Kinetoplastida: taxonomic problems and insights into the evolution of parasitism. Mem. Inst. Oswaldo Cruz, 2001, 96, 397-402.

Miles M.A., Toyé P.J., Oswald S.C. \& Godfrey D.G. The identification by isozyme patterns of two distinct strain-groups of Trypanosoma cruzi, circulating independently in a rural area of Brazil. Transaction of the Royal Society of Tropical Medicine and Hygiene, 1977, 1, 217-225.

MiLEs M.A. Transmission cycles and the heterogeneity of Trypanosoma cruzi, in: Biology of the Kinetoplastida. Lumsden W.H.R. \& Evans D.A. (Eds.), Academic Press, London, New York, San Francisco, 1979, Vol. 2., pp. 117-196.

Moreira D., López-García P. \& Vickerman K. An updated view of kinetoplastid phylogeny using environmental sequences and a closer outgroup: proposal for a new classification of the class Kinetoplastea. Int.J. Syst. Evol. Microbiol., 2004, 54, 1861-1875.

Podlipaev S.A. Insect trypanosomatids: the need to know more. Mem. Inst. Oswaldo Cruz., 2000, 95, 517-522.

Simpson A.G.B., Lukes J. \& Roger A.J. The evolutionary history of kinetoplastids and their kinetoplasts. Molecular Biology and Evolution, 2002, 19, 2071-2083.

Simpson A.G.B. \& Roger A.J. Protein phylogenies robustly resolve the deep-level relationships within Euglenozoa. Molecular Phylogenetics and Evolution, 2004, 30, 201-212.

Simpson A.G.B., Gill E.E., Callahan H.A., Litaker R.W. \& Roger A.J. Early evolution within kinetoplastids (Euglenozoa), and the late emergence of trypanosomatids. Protist, 2004, 155, 407-422.

Simpson A.G.B., Stevens J.R. \& Lukes J. The evolution and diversity of kinetoplastid flagellates. Trends in Parasitology, 2006, 22, 168-174.

Stevens J.R., Noyes H.A., Dover G.A. \& Gibson W.C. The ancient and divergent origins of the human pathogenic trypanosomes, Trypanosoma brucei and T. cruzi. Parasitology, 1999, 118, 107-116.

Stevens J.R., Noyes H.A., Schofield C.J. \& Gibson W. The mole- cular evolution of Trypanosomatidae. Advances in Parasitology, 2001, 48, 1-56.

Stothard J.R., Frame I.A., Carrasco H.J. \& Miles M.A. Temperature gradient gel electrophoresis (TGGE) analysis of riboprints from Trypanosoma cruzi. Parasitology, 1998, 117, 249-253.

VICKERMAN K. The diversity of the kinetoplastid flagellates in: Biology of the Kinetoplastida. Lumsden W.H.R. \& Evans D.A. (Eds), Academic Press, London/New York/San Francisco, 1976, 1-34.

VICKERMAN K. The evolutionary expansion of the trypanosomatid flagellates. International Journal for Parasitology, 1994, 24, 1317-1331.

Wallace F.G. The trypanosomatid parasites of insects and arachnids. Experimental Parasitology, 1966, 18, 124-193.

Woo P.T.K. On the origin of mammalian trypanosomes which develop in the anterior station of blood-sucking arthropods. Nature, 1970, 228, 1059-1062.

Wright A.D.G., Li S., Feng S., Martin D.S. \& Lynn D.H. Phylogenetic position of the kinetoplastids, Cryptobia bullocki, Cryptobia catostomi, and Cryptobia salmositica and monophyly of the genus Trypanosoma inferred from small subunit ribosomal RNA sequences. Molecular and Biochemical Parasitology, 1999, 99, 69-76. 